\title{
Complete Tunneling of Light via Local Barrier Modes in A Composite Barrier with Metamaterials
}

\author{
Kyoung-Youm Kim* \\ Department of Optical Engineering, Sejong University, Gunja-dong, Gwangjin-gu, \\ Seoul 143-747, Korea \\ Saehwa Kim \\ Information Communications Engineering Major, Hankuk University of Foreign Studies, \\ Mohyeon-myeon, Cheoin-gu, Yongin-si, Gyeonggi-do 449-791, Korea
}

(Received November 27, 2008 : accepted December 15, 2008)

\begin{abstract}
We investigate the conditions of the complete tunneling of light across a composite barrier made of multiple layers involving metamaterials. It is shown that complete tunneling phenomena are related to the resonance transmission properties of local modes formed in barrier layers and that there are two distinctive kinds of local barrier modes involved in actual complete tunneling: the degenerate inner-barrier mode and the full barrier mode. Complete tunneling occurs via two successive mode couplings: from the incident plane wave to the plane wave in the transmission layer through the direct mediation of these two kinds of local barrier modes.
\end{abstract}

Keywords: Tunneling, Metamaterials

OCIS codes : (240.7040) Tunneling; (160.3918) Metamaterials

\section{INTRODUCTION}

Complete tunneling denotes the phenomenon that the incident lights can be completely transferred across barrier layers without any loss or reflection of optical power [1-5]. We can find this somewhat surprising phenomenon in the frustrated total internal reflection (FTIR) structure shown in Fig. 1(a), where the light is incident from the incidence layer to the barrier layers having an incident angle $(\theta)$ larger than the critical angle. Similar total transmissions can be found in the Fabry-Perot resonator which adopts, for example, two metallic slabs sandwiching inner dielectric layers. In this case, however, the light fields in the inner layers can be described by sinusoidal functions that have real longitudinal wavenumbers. We can distinguish complete tunneling from such total transmissions in that longitudinal wavenumbers of the light fields in barrier layers are all imaginary. We also call the layers sandwiched between two high-index (incidence and transmission) layers barrier layers in the sense that the light

*Corresponding author: kykim@sejong.ac.kr fields there have all imaginary longitudinal wavenumbers.

In this paper, we do not impose any kind of impedancematching condition $[3,4]$ to barrier layers or any limit on the number of inner layers comprising the barrier and we will investigate the conditions for complete tunneling, especially focusing on the roles played by the local modes formed in barrier layers.

\section{THEORETICAL FRAMEWORK AND INTRODUCED ASSUMPTIONS}

We assume total $N$ barrier layers: with $n$-th layer having relative permittivity $\mathcal{E}_{n}$, relative permeability $\mu_{n}$, and length $d_{n}$. Incidence and transmission layers are assumed to be made of the same positive index medium having $\mathcal{E}_{s}$ and $\mu_{s}$. The incident light [either TE-mode $E_{y}$-field or TM-mode $H_{y}$-field $]$ can be described by $\Psi_{i}=\left[A_{0}\right.$ $\left.\exp \left(i k_{s} x\right)+B_{0} \exp \left(-i k_{s} x\right)\right] \exp \left(i \beta_{\text {inc }} z\right)$, where $k_{s}^{2}=k_{0}^{2} \epsilon_{s} \mu_{s} \cos ^{2} \theta$, $\beta_{i n c}^{2}=k_{0}^{2} \varepsilon_{s} \mu_{s} \sin ^{2} \theta$, and $k_{0}$ is the wavenumber of light in vacuum. The light fields in barrier layers are $\Psi_{n}=\left\{A_{n} \exp \right.$ $\left.\left[-\kappa_{n}\left(x-\sum_{k=1}^{n-1} d_{k}\right)\right]+B_{n} \exp \left[\kappa_{n}\left(x-\sum_{k=1}^{n} d_{k}\right)\right]\right\} \exp \left(i \beta_{\text {inc }} z\right)$, having 


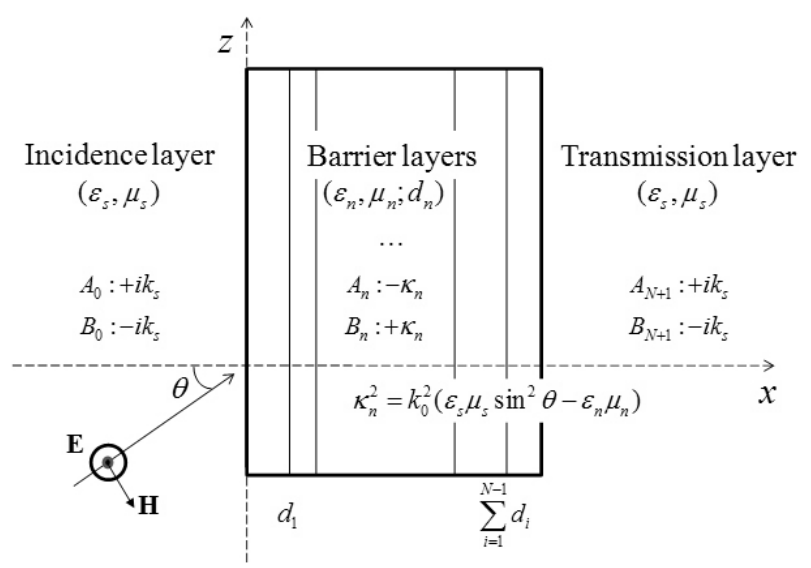

(a)

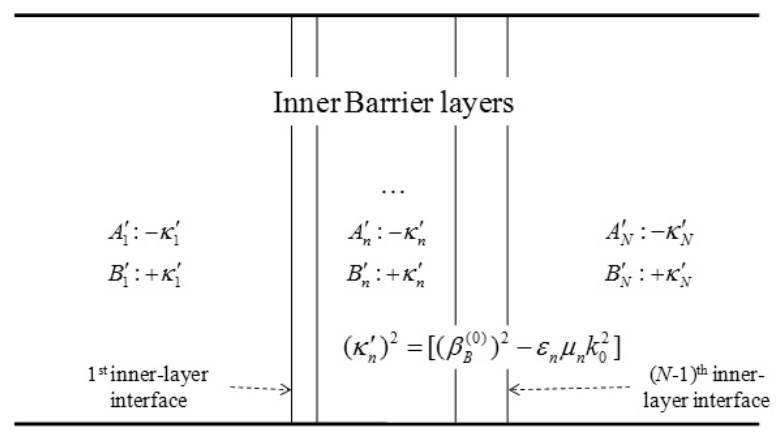

(b)

FIG. 1. (a) Tunneling of incident light across a composite barrier. (b) Barrier layers for the degenerate inner-barrier modes.

imaginary longitudinal wavenumbers $k_{n}=i \kappa_{n}=i k_{0}\left[\mathcal{E}_{s} \mu_{s} \sin ^{2}\right.$ $\left.\theta-\mathcal{E}_{\mathrm{n}} \mu_{\mathrm{n}}\right]^{1 / 2}$. In the transmission layer, it is $\Psi_{t}=\left\{A_{N+1}\right.$ $\left.\exp \left[i k_{s}\left(x-\sum_{k=1}^{N} d_{k}\right)\right]+B_{N+1} \exp \left[i k_{s}\left(x-\sum_{k=1}^{N} d_{k}\right)\right]\right\} \exp \left(i \beta_{i n c} z\right)$.

Using the transfer matrix formulation, we can obtain the following matrix equation:

$$
\left(\begin{array}{l}
A_{N+1} \\
B_{N+1}
\end{array}\right)=\mathbf{P}\left(\begin{array}{l}
A_{0} \\
B_{0}
\end{array}\right)=\mathbf{R} \cdot \mathbf{M} \cdot \mathbf{L}\left(\begin{array}{c}
A_{0} \\
B_{0}
\end{array}\right)=\mathbf{R} \cdot \Pi_{n=N-1}^{1} \mathbf{M}_{n}^{n+1} \cdot \mathbf{L}\left(\begin{array}{c}
A_{0} \\
B_{0}
\end{array}\right),
$$

where $\mathbf{L}_{l m}=0.5 \exp \left(\kappa_{1} d_{1} \delta_{l 2}\right)\left[1+(-1)^{l+m-1} i k_{s} /\left(S_{s 1} \kappa_{1}\right)\right], \quad \boldsymbol{R}_{m}=$ $\left.0.5 \exp \left[-\kappa_{N} d_{N} \delta_{m 1}\right)\right]\left[1+(-1)^{l+m-1} S_{s N} \kappa_{N} / i k_{s}\right], \quad$ and $M_{n}^{n+1} l_{l m}$ $=0.5 \quad \exp \left[\kappa_{n+1} d_{n+1} \delta_{l 2}-\kappa_{n} d_{n} \delta_{m 1}\right]\left[1+(-1)^{l+m} \kappa_{n} / S_{n, n+1} \kappa_{n+1}\right]$, where $\delta_{m n}$ is the Kronecker delta function and $S_{m n}$ is given as $\mu_{m} / \mu_{n}$ and $\varepsilon_{m} / \mathcal{E}_{n}$ for TE- and TM-mode incident lights, respectively. We can easily verify that $\mathbf{P}$ is of the form of $\left[\begin{array}{llll}P_{22}^{*} & P_{21}^{*} ; P_{21} & P_{22}\end{array}\right]$ and $\boldsymbol{M}_{n}^{n+1}$ and $\mathbf{M}$ are all real matrices. We can obtain the transmission and reflection amplitudes as $t=\operatorname{det}(\mathbf{P}) / P_{22}$ and $r=-P_{21} / P_{22}$ [6] and find conditions for complete tunneling from $P_{21}=0$.

Here, we will introduce two assumptions regarding the material and geometrical parameters of the first and last barrier layers: $\kappa_{1}= \pm \varsigma_{1 N} \kappa_{N}$ and $\kappa_{1} d_{1}=\kappa_{N} d_{N}=\Xi$.
These conditions reduce the reciprocal property of $\mathbf{P}$ (that is $t=t^{\prime}$ in $\left.\left[\begin{array}{ll}\mathrm{r}^{\prime} & 1\end{array}\right]^{T}=\mathbf{P}\left[\begin{array}{ll}0 & t^{\prime}\end{array}\right]^{T}[7]\right)$ to the condition of $|\operatorname{det}(M)|=1$, i.e., the unimodular property of $\mathbf{M}$ since we have

$$
\operatorname{det}(\mathbf{P})=\frac{\kappa_{N} \varsigma_{1 N}}{\kappa_{1} \exp \left(\kappa_{N} d_{N}-\kappa_{1} d_{1}\right)} \operatorname{det}(\mathbf{M})
$$

We will now investigate separately the conditions for complete tunneling under each case of these assumptions.

\section{CASE I: $\kappa_{1}=-\varsigma_{1 N} \kappa_{N}$ and $\kappa_{1} d_{1}=\kappa_{N} d_{N}=\Xi$}

\section{A. Mathematical conditions for complete tunneling}

Above assumptions reduce the above reciprocity condition to $\operatorname{det}(\mathbf{M})=-1$ and entail $\varsigma_{1 N}<0$, which means that the incident light feels the first and last barrier layers as different kinds of metamaterials. That is, if the incident light is $\mathrm{TM}(\mathrm{TE})$-polarized, permittivity (permeability) values of the first and last barrier layers must have opposite signs (e.g., negative index-positive index or epsilon negative-mu negative medium pair can be adopted for them). After some algebra, we can obtain $P_{21}=0.25[\Gamma+i \Omega]$ where

$$
\begin{aligned}
\Gamma & =2\left[M_{22} \exp (\Xi)+M_{11} \exp (-\Xi)\right], \\
\Omega & =\left(k_{s} / \varsigma_{s 1} \kappa_{1}-\varsigma_{s 1} \kappa_{1} / k_{s}\right)\left[M_{22} \exp (\Xi)-M_{11} \exp (-\Xi)\right] \\
& +\left(k_{s} / \varsigma_{s 1} \kappa_{1}+\varsigma_{s 1} \kappa_{1} / k_{s}\right)\left(M_{12}-M_{21}\right),
\end{aligned}
$$

and from $P_{21}=0$, we have for complete tunneling

$$
\begin{aligned}
& M_{22}=-M_{11} \exp (-2 \Xi), \\
& M_{12}-M_{21}=2 M_{11} \exp (-\Xi) \frac{k_{s}^{2}-\zeta_{s}^{2} \kappa_{1}^{2}}{k_{s}^{2}+\zeta_{s 1}^{2} \kappa_{1}^{2}} .
\end{aligned}
$$

We can see that if $M_{22}=0$, eqs. (5) and (6) reduce to $\mathbf{M}= \pm\left[\begin{array}{lll}0 & 1 ; 1 & 0\end{array}\right]$ (recall that $\mathbf{M}$ is a real matrix). If we substitute this in eq. (1), we can obtain $t= \pm 1$. Therefore, if (1) $M_{11}=M_{22}=0$ and (2) $M_{12}=M_{21}= \pm 1$, complete tunneling can occur.

We note that we have simplified the problem of finding appropriate solutions of eqs. (5) and (6) by considering only a very special case of $M_{22}=0$. This reduces the transfer matrix $\mathbf{M}$ to a very trivial one and considerably limits the properties of inner barrier layers.

\section{B. Physical meanings for the complete tunneling conditions}

(1) $M_{22}=0$ and $M_{11}=0$ are the dispersion relations of two mathematical eigenmodes considering only the inner barrier structure, i.e., inner barrier layers only assuming the lengths of the first and last barrier layers to be infinite [see Fig. 1(b)] [6]. The former is for the physical 
eigenmode which has $A^{\prime}{ }_{1}=B^{\prime}{ }_{N}=0$, i.e., which has vanishing light intensities at the barrier boundaries (which are actually located at infinities), while the latter is for the unphysical eigenmode which has infinite light intensities at those boundaries, i.e., $A_{N}^{\prime}=B^{\prime}{ }_{1}=0$. We will call these modes physical and unphysical inner-barrier modes, respectively. Therefore, the condition (1) requires the existence of degenerate physical and unphysical eigenmodes. Such an eigenmode can exist when its field distribution in every inner barrier layer consists of only one of exponentially increasing and exponentially decreasing terms, i.e., such a mode can be expressed mathematically in the form of $\exp \left( \pm \kappa_{n}{ }^{\prime} x\right) \exp \left(i \beta_{B}^{(0)} z\right)$, where $\kappa_{n}{ }^{\prime}=\left[\left(\beta_{B}^{(0)}\right)^{2}-\mathcal{E}_{n} \mu_{n} k_{0}^{2}\right]^{1 / 2}$ and $\beta_{B}^{(0)}$ is the propagation constant along the $z$ axis. We can easily verify that we can obtain such degenerate eigenmodes when $\kappa_{n}{ }^{\prime}= \pm \mathrm{s}$

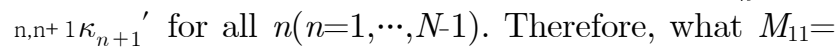
$M_{22}$ requires for complete tunneling is $\kappa_{n}=\kappa_{n}{ }^{\prime}$, resulting in a kind of phase matching condition between the incident light and the degenerate inner-barrier modes, i.e., $\beta_{\text {inc }}=k_{0}\left(\varepsilon_{\mathrm{s}} \mu_{\mathrm{s}}\right)^{1 / 2} \sin \theta_{\mathrm{CT}}=\beta_{B}^{(0)}$, where $\theta_{\mathrm{CT}}$ denotes the specific incident angle at which complete tunneling occurs.

(2) $M_{12}=M_{21}= \pm 1$ under the condition (1) means that the field intensities of the inner-barrier modes at the first and last [( $N-1)$-th] inner-layer interfaces [see Fig. 1(b)] become the same. If $M_{12}=M_{21}=1$, then the fields at the first and last inner-layer interfaces become in phase while they are out of phase when $M_{12}=M_{21}=-1$.

Summarizing the above discussions, in addition to $\kappa_{1}$ $=-\mathrm{S}_{1 N} \kappa_{\mathrm{N}}$ and $\kappa_{1} d_{1}=\kappa_{N} d_{N}=\Xi$, we need only one more necessary condition for complete tunneling: the phase matching between the incident light and the degenerate innerbarrier modes that have the same field intensities at the first and last inner-layer interfaces. Only when there exist such degenerate modes, the incident plane waves can be completely decomposed and coupled to them (the amount of each can be determined by the matrix $\mathbf{L}$ ). Then, we can say that complete tunneling occurs via two paths of mode couplings: from the incident plane wave to the (1) physical and (2) unphysical inner-barrier modes, then finally to the plane wave in the transmission layer.

\section{CASE II: $\kappa_{1}=\varsigma_{1 N} \kappa_{N}$ and $\kappa_{1} d_{1}=\kappa_{N} d_{N}=\Xi$}

\section{A. Mathematical conditions for complete tunneling}

Above assumptions reduce the reciprocity condition to $\operatorname{det}(\mathbf{M})=1$, which implies the reciprocity in light transmissions through the inner barrier layers. These assumptions entail $\varsigma_{1 N}>0$, which indicates that the incident light feels the first and last barrier layers as the same kind of metamaterials. That is, if the incident light is TE-polarized, permeability values of the first and last barrier layers must have the same sign.

Similarly, we have $P_{21}=0.25[\Gamma+i \Omega]$ where

$$
\begin{aligned}
\Gamma & =2\left(M_{12}+M_{21}\right), \\
\Omega & =\left(k_{s} / \varsigma_{s 1} \kappa_{1}+\varsigma_{s 1} \kappa_{1} / k_{s}\right)\left[M_{22} \exp (\Xi)-M_{11} \exp (-\Xi)\right] \\
& +\left(k_{s} / \varsigma_{s 1} \kappa_{1}-\varsigma_{s 1} \kappa_{1} / k_{s}\right)\left(M_{12}-M_{21}\right) .
\end{aligned}
$$

Therefore, we have from $P_{21}=0$

$$
\begin{aligned}
& M_{12}=-M_{21}, \\
& M_{22} \exp (\Xi)-M_{11} \exp (-\Xi)=2 M_{21} \frac{k_{s}^{2}-\varsigma_{s 1}^{2} \kappa_{1}^{2}}{k_{s}^{2}+\zeta_{s 1}^{2} \kappa_{1}^{2}}
\end{aligned}
$$

Eq. (9) and the unimodular property of $\mathbf{M}$ make $t^{\prime}=t^{\prime \prime}$ and $r^{\prime}=r^{\prime \prime}$ in the following two matrix equations: $\left[\mathrm{t}^{\prime}\right.$ $0]^{T}=\mathbf{M}\left[\begin{array}{ll}1 & r^{\prime}\end{array}\right]^{T}$ and $\left[\begin{array}{ll}r^{\prime \prime} & 1\end{array}\right]^{T}=\mathbf{M}\left[\begin{array}{ll}0 & t^{\prime \prime}\end{array}\right]^{T}$, which suggest that the barrier must have a symmetric inner layer structure $[6,7]$ for complete tunneling, which entails $\kappa_{1}=\kappa_{N}$ and $d_{1}=d_{N}$.

\section{B. Physical meanings for the complete tunneling conditions}

Now, let us investigate the radiation modes of the symmetric composite barrier and then relate them to eq. (10). Radiation modes of the tunneling barrier have real longitudinal wavenumbers $\left(k_{\text {rad }}\right)$ and form standing waves in the incidence and transmission layers, allowing no power flow or leakage along that direction [8], while they have imaginary wavenumbers given by $i \kappa_{n}^{\prime \prime}=i\left[\beta_{B}^{2}\right.$ - $\varepsilon$ $\left.{ }_{n} \mu_{n} k_{0}^{2}\right]^{1 / 2}$ in the barrier layers, where $\beta_{B}$ denotes their real propagation constant $\left(k_{\text {rad }}^{2}+\beta_{B}^{2}=\mathcal{E}_{s} \mu_{s} k_{0}^{2}\right)$ along the transverse $(z)$ direction. A continuum of propagation constants can be found using the following dispersion relation

$\left(\begin{array}{c}\exp \left(i \phi_{t}\right) \\ \exp \left(-i \phi_{t}\right)\end{array}\right)=\mathbf{P}\left(\begin{array}{c}\exp \left(i \phi_{i}\right) \\ \exp \left(-i \phi_{i}\right)\end{array}\right)=\left(\begin{array}{cc}P_{22}^{*} & -i\left|P_{21}\right| \\ i\left|P_{21}\right| & P_{22}\end{array}\right)\left(\begin{array}{c}\exp \left(i \phi_{i}\right) \\ \exp \left(-i \phi_{i}\right)\end{array}\right)$

where $P_{21}$ becomes pure imaginary due to the symmetry of the barrier structure $\left(P_{21}^{*}=-P_{21}\right)$. In eq. (11), we assumed $\varphi_{i}=2 \cos \left(\mathrm{k}_{\text {rad }} x+\phi_{i}\right) \exp \left(i \beta_{B} z\right)$ and $\varphi_{t}=2 \cos \left[\mathrm{k}_{\text {rad }}\right.$ $\left.\left(x-\sum_{k=1}^{N} d_{k}\right)+\phi_{t}\right] \exp \left(i \beta_{B} z\right)$, in the incidence and transmission layers, respectively, where $\phi_{i}$ denotes an arbitrary phase factor of the standing wave in the incidence layer. Here, we impose a restraint on the continuum of radiation modes: the phase difference between the standing waves in the incidence and transmission layers $\left(\phi_{i}-\right.$ $\phi_{t}$ ) must remain constant regardless of the arbitrary phase factor $\phi_{i}$. This constraint gives us, after some algebra, $P_{21}=0$ and $\left|P_{22}\right|^{2}=1$. Therefore, we can see that eq. (10) describes the dispersion relation of a special kind of radiation modes. If we set $\Xi \rightarrow \infty$ in eq. (10), 


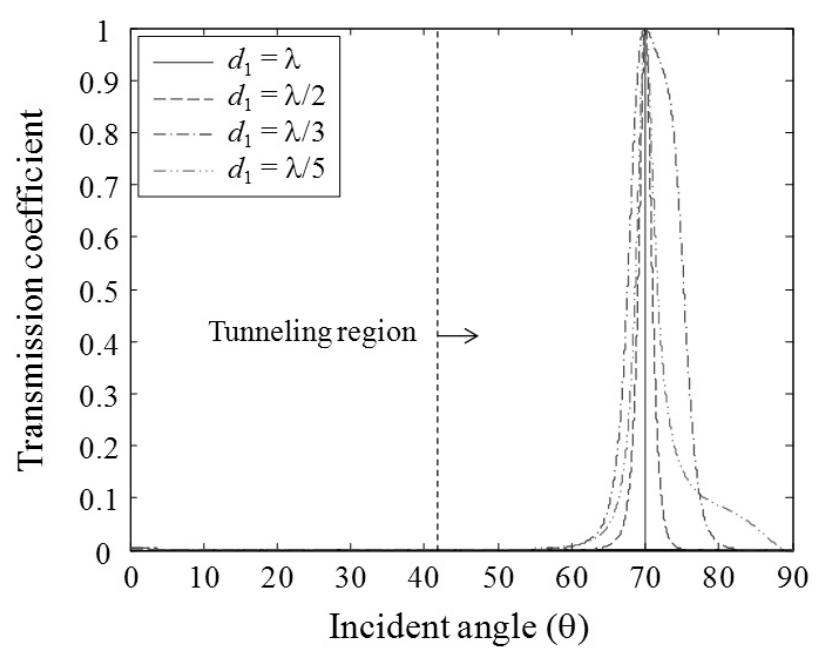

FIG. 2. Effect of the lengths of the first and last barrier layers on the transmission coefficients of the TM incident light through a FTIR structure whose incident and transmission layers are made of glass. Material and geometrical parameters of the composite tunneling barrier are as follows: $\varepsilon_{1}=1, \quad \varepsilon_{2}=-2.5, \quad \varepsilon_{3}=1.667, \quad \varepsilon_{4}=0.926, \quad \varepsilon$ ${ }_{5}=-0.772, \mu_{1}=1, \mu_{2}=1.672, \mu_{3}=-0.453, \mu_{4}=1.232, \mu_{5}=-1.813$ and $d_{2}=0.274 \lambda, d_{3}=0.3 \lambda, d_{4}=0.2 \lambda, d_{5}=\kappa_{1} d_{1} / \kappa_{5}$. Since $S$ ${ }_{15}=-1.296$, this composite barrier corresponds to case $I$.

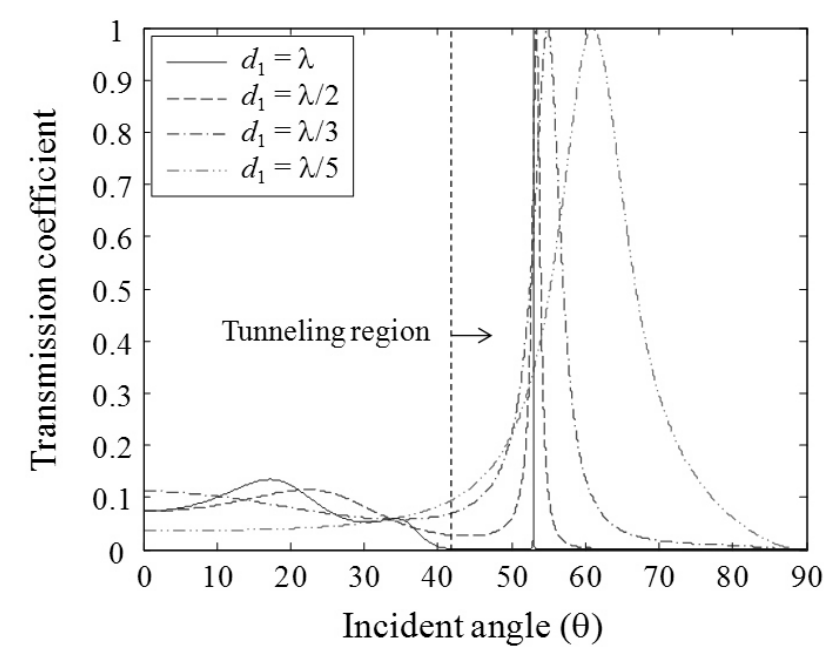

FIG. 3. The same as Fig. 2 with the following parameters: $\mathcal{E}_{1}=\mathcal{E}_{5}=1, \varepsilon_{2}=\varepsilon_{4}=-2.5, \mathcal{E}_{3}=1.25, \mu_{1}=\mu_{2}=\mu_{3}=\mu_{4}=\mu_{5}=1$, and $d_{2}=d_{3}=d_{4}=0.1 \lambda, d_{5}=d_{1}$. Since $\varsigma_{15}=1$ and the barrier is center-symmetric, this composite barrier corresponds to case II.

we can obtain a simple equation of $M_{22} \rightarrow 0$ (but not necessarily $M_{11} \rightarrow 0$, which is a different characteristic compared with case I), and this is, as has been mentioned above, the dispersion relation of the physical inner-barrier modes. Therefore, we can see that as the lengths of the first and last barrier layers become large, the radiation modes described by eq. (10) converge on the physical inner-barrier modes mentioned above. From this fact, we can re-interpret that eq. (10) corresponds to the dispersion relation of physical inner-barrier modes considering the finite barrier effects, i.e., including the influences of the finite lengths of the first and last barrier layers, and the presence of incidence and transmission layers. From this respect, we call them the full barrier modes. Therefore, what eq. (10) demands for complete tunneling is $\kappa_{n}=\kappa_{n}^{\prime \prime}$ or $k_{s}=k_{\text {rad }}$, resulting similarly in the phase matching condition between the incident light and the full barrier modes: $\beta_{\mathrm{inc}}=k_{0}\left(\mathcal{E}_{\mathrm{s}} \mu_{\mathrm{s}}\right)^{1 / 2} \sin \theta_{C T}=\beta_{B}$.

Summarizing above discussions, in case of $\kappa_{1}=\varsigma_{1 N} \varsigma_{N}$ and $\kappa_{1} d_{1}=\kappa_{N} d_{N}=\Xi$, we need two more necessary conditions for complete tunneling: the center-symmetric property of the composite tunneling barrier structure and the phase matching between the incident light and the full barrier modes. Such full barrier modes are different from the physical inner-barrier modes due to the influence of the finite lengths of the first and last barrier layers and the presence of incidence and transmission layers.

\section{NUMERICAL CALCULATIONS}

For a proof-of-principle example, we calculated the transmission coefficients of TM-mode incident light $(\lambda$ $=1550 \mathrm{~nm}$ ) through two FTIR structures having 5 inner layers, one corresponding to (a) case $I$ and (b) case II. We changed only the lengths of the first and last barrier layers, while all the other material and geometrical parameters remain the same, their detailed values shown in figure captions. The results are plotted in Figs. 2 and 3, respectively. In Fig. 2, $\theta_{C T}$ remains constant for all $d_{1}$ (and $d_{5}$ ) values, while it is changed by them in Fig. 3. This contrasting feature is due to the different kind of local barrier modes actually involved in complete tunneling. In case $I, \theta_{C T}$ 's are determined by the propagation constants of degenerate inner-barrier modes, which are not affected by the lengths of the first and last barrier layers while, in case II, they are determined by those of full barrier modes, which include the effects of the finite lengths of the first and last barrier layers and the presence of incidence and transmission layers. Especially, we can confirm that as the lengths of the first and last barrier layers increase, $\theta_{C T}$ 's converge on the specific value at which the incident light satisfies the phase matching condition with the physical inner-barrier mode.

\section{CONCLUSION}

We found the conditions for the complete tunneling of light across a composite barrier made of multiple layers involving metamaterials. Especially, we explicitly showed that one necessary condition for complete tun- 
neling is the phase matching between the incident light and the local barrier modes, and there are two different kinds of such local barrier modes: the degenerate innerbarrier modes and the full barrier modes. Complete tunneling results from the complete transfer of the incident optical power to the transmission layer through the direct mediation of these two kinds of local barrier modes.

\section{ACKNOWLEDGMENT}

S. Kim acknowledges the support by Hankuk University of Foreign Studies Research Fund of 2008.

\section{REFERENCES}

[1] Z. M. Zhang and C. J. Fu, "Unusual photon tunneling in the presence of a layer with a negative refractive index," Appl. Phys. Lett., vol. 80, no. 6, pp. 1097-1099, 2002.

[2] A. Alù and N. Engheta, "Pairing an epsilon-negative slab with a mu-negative slab: resonance, tunneling and transparency," IEEE Trans. Antennas Propag., vol. 51, no. 10, pp. 2558-2571, 2003.

[3] K.-Y. Kim, "Photon tunneling in composite layers of negative-and positive-index media," Phys. Rev. E, vol. 70, no. 4, Article 047603, 2004.

[4] K.-Y. Kim, "Properties of photon tunneling through singlenegative materials," Opt. Lett., vol. 30, no. 4, pp. 430432, 2005.

[5] X. Zhou and G. Hu, "Total transmission condition for photon tunnelling in a layered structure with metamaterials," J. Opt. A, vol. 9, no. 1, pp. 60-65, 2007.

[6] E. Anemogiannis, E. N. Glytsis, and T. K. Gaylord, "Determination of guided and leaky modes in lossless and lossy planar multilayer optical waveguides: reflection pole method andwavevector density method," IEEE J. Lightwave Tech., vol. 17, no. 5, pp. 929-941, 1999.

[7] R. J. Potton, "Reciprocity in optics," Rep. Prog. Phys., vol. 67, no. 5, pp. 717-754, 2004.

[8] S.-L. Lee, Y. Chung, L. A. Coldren, and N. Dagli, "On leaky mode approximations for modal expansion in multilayer openwaveguides," IEEE J. Quantum Electron., vol. 31, no. 10, pp. 1790-1802, 1995. 